\section{Data-dependent acquisition}

B. Güssregen

Merck KGaA, Darmstadt, Deutschland

Synonym(e) Data-directed analysis; DDA Information-Dependent Acquisition; Smart select

Englischer Begriff information-dependent acquisition; data directed analysis; smart select

Definition Methode in der $\triangleright$ Massenspektrometrie, bei der in Abhängigkeit eines Meßergebnisses „on the fly“ ein weiteres Experiment durchgeführt wird.
Beschreibung Ein IDA-Experiment besteht aus einem „Survey"-Scan und ein oder mehreren „Dependent"-Scans. Tritt ein im „Survey“-Scan programmiertes Ereignis ein, z. B. ein Ionenübergang in einem MRM-Experiment, triggert dieses softwaregesteuert einen Wechsel in einen anderen Messmodus, wie z. B. Produkt-Ionen-Scan. Anschließend wird wieder in den vorherigen Messmodus gewechselt.

\section{Literatur}

Niessen WMA (2006) Liquid chromatography-mass spectrometry. CRC Press, New York 